\title{
Efeitos da irradiação com raios- $X$ em anastomoses colônicas: estudo estereológico experimental em ratos 1
}

Effects of X-rays irradiation on colonic anastomoses: experimental stereological study in rats

\author{
Francisco Lopes-Paulo ${ }^{2}$
}

1. Trabalho realizado no Laboratório de Cirurgia Experimental da Faculdade de Ciências Médicas e no Departamento de Anatomia Humana do Instituto Biologia da Universidade Estadual do Rio de Janeiro (UERJ).

2. Professor Titular de Cirurgia Colorretal da Faculdade de Ciências Médicas da UERJ.

\section{RESUMO}

OBJETIVO: Este estudo foi realizado para quantificar possíveis alterações na tensão de ruptura e na concentração de colágeno em anastomoses realizadas em cólon irradiado.

MÉTODOS: Doze ratos Wistar foram submetidos a secção e anastomose do cólon, constituindo dois grupos: $A n$ (controle, $\mathrm{n}=7$ ) e $I+A n$ (irradiado, $\mathrm{n}=5$ ). $\mathrm{O}$ grupo $A n$ foi submetido apenas à cirurgia enquanto o grupo I+An recebeu dose única de irradiação (1096 cGy) 7 dias antes da operação. Todos foram sacrificados no sétimo dia pós-operatório, para verificação da tensão de ruptura da anastomose e quantificação do colágeno. A quantificação do colágeno foi feita por estereologia de cortes verticais.

RESULTADOS: $O$ grupo $A n$ apresentou valores superiores ao grupo $I+A n$ quando comparados a pressão de ruptura da anastomose (172 mm Hg X 60 mm Hg, p = 0,004) e o índice de colágeno (14,24 X 1,62, $\mathrm{p}=0,004)$. No grupo An o local de ruptura do cólon ocorreu com maior freqüência na área perianastomótica, enquanto que no grupo $I+A n$ a ruptura ocorreu com maior freqüência na linha anastomótica $(p=0,007)$.

CONCLUSÃO: A irradiação prejudicou a cicatrização anastomótica, conforme evidenciado pela diminuição da pressão de ruptura da anastomose e do índice de colágeno no grupo de animais irradiados.

DESCRITORES: Cólon. Irradiação. Anastomose. Deiscência. Tensão de ruptura. Colágeno.

\begin{abstract}
PURPOSE: This study was done in order to quantify changes on bursting pressure and collagen content on irradiated colon anastomosis.

METHODS: Twelve Wistar rats were submitted to colon section and anastomosis. Animals were divided in two groups: $A n$ (control, $\mathrm{n}=7$ ) and $I+A n$ (irradiated, $\mathrm{n}=5$ ). An group was submitted only to surgery while $I+A n$ received a single dose irradiation (1096 cGy) seven days before operation. Animals were sacrificed on seventh postoperative day to quantify anastomosis bursting pressure and collagen content. Collagen quantification was done by vertical section stereology.

RESULTS: An group present higher values of anastomosis bursting pressure (172 mm Hg X $60 \mathrm{~mm} \mathrm{Hg}, \mathrm{p}$ $=0,004)$ and collagen content $(14,24 X 1,62, p=0,004)$ as compared to $I+A n$ group. On An group the site of colon rupture was more frequently found on the perianastomotic area while on $I+A n$ group the rupture was mainly on anastomotic line $(\mathrm{p}=0,007)$.
\end{abstract}

CONCLUSION: Irradiation impaired anastomotic healing as evidenced by reduction of anastomotic bursting 
pressure and collagen content on irradiated animals.

KEY WORDS: Colon. Irradiation. Anastomosis. Leakage. Bursting pressure. Collagen.

\section{Introdução}

As lesões actínicas do tubo digestivo constituem um dos problemas mais desafiadores com que o cirurgião colorretal pode se defrontar. Suas manifestações podem ser precoces, durante o tratamento, ou surgirem anos após o emprego da radioterapia. O dano tecidual causado pela radiação é cumulativo e progressivo, existindo uma dose limite, além da qual qualquer dose adicional, aplicada a qualquer tempo após o tratamento inicial, pode precipitar as complicações. Certas patologias associadas, tais como o diabetes melitus, hipertensão arterial e cirurgia abdominal prévia podem predispor o paciente à enterite actínica ${ }^{1}$.

Existe controvérsia na literatura a respeito dos riscos potenciais de deiscência da anastomose após radioterapia pré-operatória ${ }^{2}$. Rider col. ${ }^{3}$ relataram que não houve diferença significativa no número de complicações pós-operatórias entre um grupo controle e outro que recebeu radioterapia préoperatória. Porém Stevens e col. ${ }^{4}$ relataram aumento da freqüência de deiscência de anastomoses colorretais baixas em pacientes submetidos a radioterapia pré-operatória, quando comparada com um grupo controle, e recomendou a proteção da anastomose com uma colostomia temporária. Baseado nessas controvérsias, este estudo experimental foi realizado para quantificar possíveis alterações na tensão de ruptura de anastomoses realizadas em cólon irradiado assim como na concentração de colágeno, já que sua síntese é essencial no processo de cicatrização ${ }^{5}$.

\section{Métodos}

\section{Amostra}

Foram utilizados 12 ratos adultos da raça Wistar, do sexo masculino, cujo peso médio era de 294,41 g (mín=242g; máx=360g). Esses animais foram divididos em dois grupos de estudo, sem diferença significativa de peso entre os mesmos $(p=0,12)$, com a seguinte formação: grupo controle $(A n)(n=7)$ e grupo irradiado $(I+A n)(\mathrm{n}=5)$. O grupo $A n$ foi submetido a uma anastomose colônica, enquanto o grupo $I+A n$ recebeu irradiação abdominal e uma anastomose colônica sete dias após. Os animais de ambos os grupos tiveram livre acesso à água e ração comercial (Nuvolab ${ }^{\circledR}$ CR1) durante toda a experimentação e foram mantidos em gaiolas individuais, com períodos de luz de 07 às 19 horas.

\section{Procedimentos}

\section{Anastomose colônica}

Os animais foram operados sob anestesia geral (tiopental sódico, $50 \mathrm{mg} / \mathrm{kg}$ / peso intraperitoneal). $\mathrm{O}$ acesso à cavidade abdominal foi feito por laparotomia mediana, com 2 cm de extensão, eqüidistante do gradil costal e da borda superior da pelve. Em seguida foi introduzido um catéter peridural 20 G (Becton-Dickinson), pelo orifício anal, com 8 cm de extensão, sendo o cólon seccionado transversalmente à altura de sua extremidade. Em seguida foi realizada anastomose término-terminal evertida, com 8 pontos separados de Dexon ${ }^{\circledR}$ 5-0 (Davis+Geck), englobando todas as camadas da parede intestinal. A laparotomia foi fechada por planos, com sutura contínua de Prolene ${ }^{\circledR}$ 5-0.

\section{Irradiacão}

Os animais do grupo $I+A n$ foram imobilizados em caixas plásticas e receberam dose única de irradiação, de 1096 cGy, no primeiro dia da experimentação, liberados a partir de um acelerador linear de $06 \mathrm{MeV}$, modelo Mevatron ${ }^{\circledR}$ (Siemens), a uma taxa de liberação de 137 cGy por minuto, com distância fonte-pele de $100 \mathrm{~cm}$, aplicados sobre o abdome, em um campo de 6 X 4 cm.

\section{Reoperação}

Os animais de ambos os grupos foram mantidos em condições ambientais e de alimentação idênticas, tendo sido sacrificados no sétimo dia de pósoperatório. Foram obtidos segmentos colônicos de 
seis centímetros de extensão, contendo a anastomose em sua porção mediana.

\section{Pressão de ruptura da anastomose}

Os segmentos colônicos obtidos de cada animal foram lavados com soro fisiológico para remoção de resíduos fecais. Em seguida a extremidade distal foi ocluída, enquanto a proximal recebeu um catéter de polietileno que era conectado a um divisor de fluxo tipo “T”. Um dos ramos desse conector era ligado a um compressor, que liberava um fluxo de ar de 0,5 litro por minuto. O outro ramo era conectado a um transdutor, por sua vez ligado a um polígrafo modelo Polygraph $2006^{\circledR}$ (Letica Scientific Instruments, Espanha), que registrava a curva ascendente de pressão no interior do segmento colônico até o momento de sua ruptura (PR). Nesse momento era registrado também o local dessa ruptura, que poderia ocorrer na linha anastomótica, na alça aferente ou na alça eferente.

\section{Processamento histológico}

Após a medida da pressão de ruptura, os segmentos colônicos foram clivados e processados para inclusão em parafina, de acordo com as normas para análise por estereologia de cortes verticais ${ }^{6-8}$. Em seguida foram obtidos cortes com $5 \mu \mathrm{m}$ de espessura que foram corados pelo picro sirius red para evidenciação do colágeno.

\section{Análise estereológica}

Os cortes histológicos foram analisados através de um sistema microscópico computadorizado, composto por um microscópio óptico Olympus BH2, dotado de objetivas plano-acromáticas. A ocular era acoplada a uma câmera de vídeo marca Galai, que transmitia as imagens obtidas a um microcomputador, modelo Pentium-III de $800 \mathrm{Mhz}$, com 256 megabytes de memória RAM. Esse computador exibia as imagens em um monitor de 17 polegadas, com dot pitch 0,23 e aumento final de até $200 \mathrm{X}$. As imagens assim transmitidas foram capturadas e tratadas através do programa Adobe Photoshop $^{\circledR}$, versão 7.0.1. Foram analisadas duas imagens histológicas de campos aleatórios em cada corte, totalizando quatro imagens por animal. A coloração histológica com o picro sirius red, marca em vermelho o colágeno. A observação dessas lâminas com o auxílio de um filtro analisador combinado a um filtro polarizador, interpostos ao sistema microscópico óptico, geram imagens com fundo negro, ressaltando as fibras colágenas coradas em tom vermelho intenso (Figuras 1 e 2).

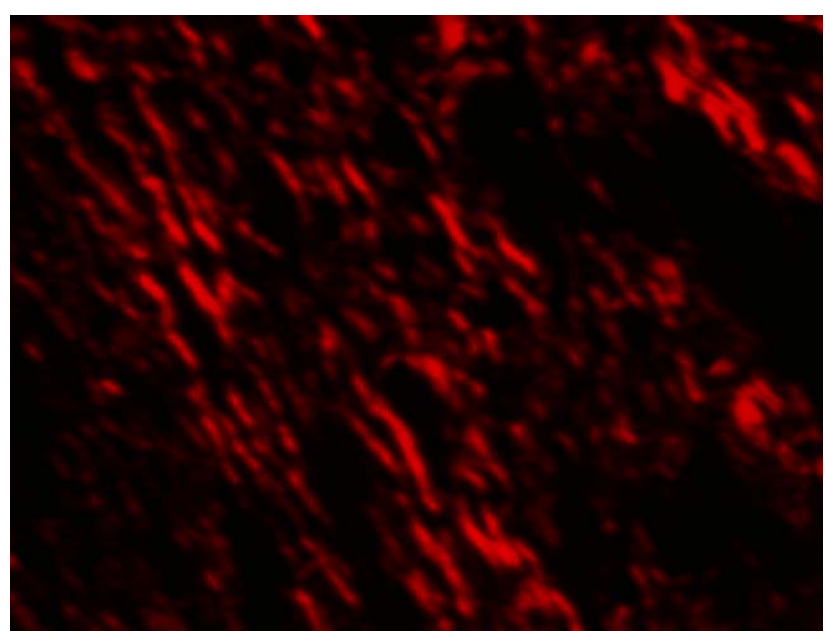

FIGURA 1 - Imagem de corte histológico da área de anastomose em animal do grupo An, corado pelo picro sirius red e observado com filtros analisador e polarizador (200x).

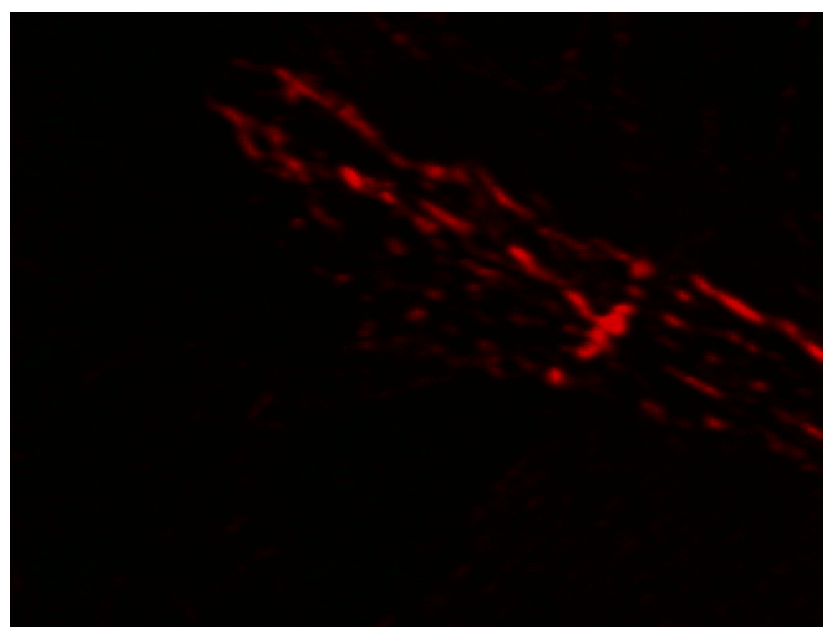

FIGURA 2 - Imagem de corte histológico da área de anastomose em animal do grupo I+An, corado pelo picro sirius red e observado com filtros analisador e polarizador (200x).

As imagens assim obtidas e capturadas pelo computador, permitem que o software calcule a média das intensidades dos pixels corados em vermelho, numa escala de 0 a 255, aqui denominado índice de colágeno (Ic), permitindo a tradução numérica do colágeno existente em cada corte, para comparação entre os grupos de estudo ${ }^{(9)}$. 


\section{Análise estatística}

A análise estatística do peso, pressão de ruptura da anastomose e índice de colágeno foi feita pelo teste de Mann-Whitney, enquanto o local de ruptura foi analisado pelo Teste Exato de Fisher. Em todas as análises, um valor de $\mathrm{p} \geq 0,05$ ou $5 \%$ foi estabelecido para rejeição da hipótese nula.

Este estudo foi realizado no Laboratório de Cirurgia Experimental da Faculdade de Ciências Médicas da Universidade do Estado do Rio de Janeiro, de acordo com as normas técnicas e éticas desse laboratório. O processamento histológico e a análise estereológica foram realizadas no Departamento de Anatomia do Instituto de Biologia da UERJ.

\section{Resultados}

Os dados obtidos nos grupos de estudo estão representados nas Tabelas 1 e 2.

A pressão de ruptura da parede colônica no grupo An variou $156 \mathrm{~mm} \mathrm{Hg}$ a $190 \mathrm{~mm} \mathrm{Hg}$, com mediana de $172 \mathrm{~mm}$ Hg. No grupo $I+A n$ a pressão variou de $20 \mathrm{~mm} \mathrm{Hg}$ a $72 \mathrm{~mm} \mathrm{Hg}$, com mediana de $60 \mathrm{~mm} \mathrm{Hg}$, sendo a diferença entre ambos estatisticamente significativa ( $\mathrm{p}=0,004)$ (Figura 3).

No grupo An, apenas um animal apresentou ruptura na linha de anastomose. Os demais apresentaram ruptura à montante ou à jusante da linha anastomótica. Esse resultado contrastou com o grupo $I+A n$, no qual todas as rupturas foram na linha de anastomose $(\mathrm{p}=0,007)$ (Tabela 3$)$.

O índice de colágeno no grupo An apresentou mediana de 14,24, com mínimo de 11,74 e máximo de 24,19. No grupo $I+A n$ esse índice foi menor que no grupo $A n$, apresentando mediana de 1,62, com mínimo de 0,85 e máximo de $3,51(\mathrm{p}=0,004)$ (Figura 4).

O índice de colágeno (Ic) representa uma medida da intensidade média de brilho dos pixels vermelhos das imagens aferido pelo canal vermelho do histograma da imagem. No grupo An o conteúdo de colágeno foi avaliado por essa técnica e também pela superposição de uma grade estereológica às imagens, que fornece o volume parcial do colágeno ( $V v)$ em números percentuais. Comparando-se os dados obtidos pelas duas técnicas, imagem por imagem nesse grupo e aplicando-se o teste de correlação de Pearson, foi encontrada uma forte correlação positiva
TABELA 1 - Dados obtidos no grupo An.

\begin{tabular}{|c|c|c|c|}
\hline Rato & $\begin{array}{c}\text { Peso } \\
\text { (g) }\end{array}$ & $\begin{array}{c}\text { PR } \\
\text { (mm Hg) }\end{array}$ & Ic \\
\hline $\mathbf{1}$ & 266 & 190 & 24,19 \\
\hline $\mathbf{2}$ & 300 & 198 & 13,41 \\
\hline $\mathbf{3}$ & 296 & 176 & 13,41 \\
\hline $\mathbf{4}$ & 256 & 178 & 15,21 \\
\hline $\mathbf{5}$ & 264 & 158 & 11,74 \\
\hline $\mathbf{6}$ & 242 & 156 & 17,34 \\
\hline $\mathbf{7}$ & 242 & 162 & 14,24 \\
\hline
\end{tabular}

PR = pressão de ruptura, Ic = Índice de colágeno

TABELA 2 - Dados obtidos no grupo I+An.

\begin{tabular}{|c|c|c|c|}
\hline Rato & $\begin{array}{c}\text { Peso } \\
\text { (g) }\end{array}$ & $\begin{array}{c}\text { PR } \\
\text { (mm Hg) }\end{array}$ & Ic \\
\hline $\mathbf{1}$ & 360 & 60 & 3,51 \\
\hline $\mathbf{2}$ & 308 & 72 & 1,63 \\
\hline $\mathbf{3}$ & 262 & 60 & 1,12 \\
\hline $\mathbf{4}$ & 288 & 48 & 0,85 \\
\hline $\mathbf{5}$ & 290 & 20 & 3,50 \\
\hline
\end{tabular}

$\mathrm{PR}=$ pressão de ruptura, Ic = Índice de colágeno

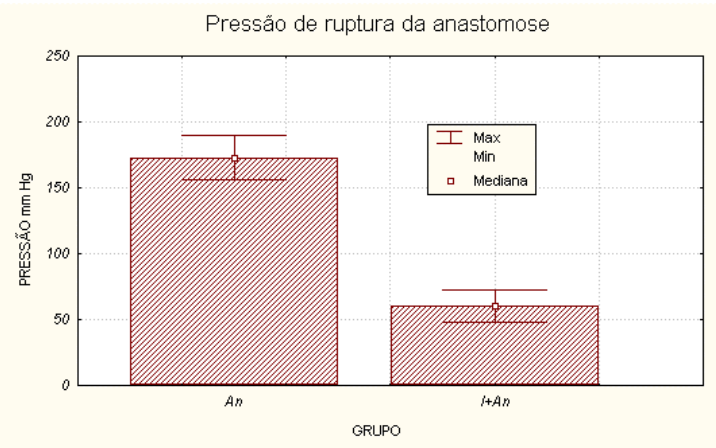

FIGURA 3 - Variação da pressão de ruptura da anastomose nos grupos estudados $(p=0,004)$.

TABELA 3 - Local de ruptura da parede colônica.

\begin{tabular}{|l|c|c|}
\hline Grupo & LA & PA \\
\hline An & 1 & 6 \\
\hline I+An & 5 & 0 \\
\hline
\end{tabular}

$\mathrm{LA}=$ ruptura na linha anastomótica ; $\mathrm{PA}=$ ruptura perianastomótica ( $\mathrm{p}=0,007)$. 


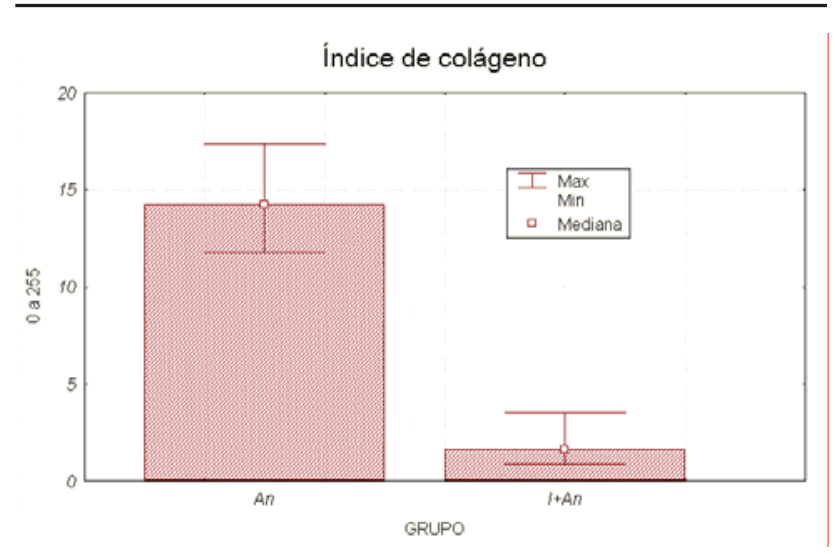

FIGURA 4 - Variação do índice de colágeno nos grupos estudados $(p=0,004)$.

entre as duas medidas $(r=0,98)$. Por isso optou-se pela medida direta do índice de colágeno, por sua maior praticidade e rapidez. O volume parcial de colágeno pode ser calculado a partir do índice pela fórmula: $\mathrm{Vv}=4,5601+1,1836$. Ic.

\section{Discussão}

A irradiação dos animais deste estudo foi feita em dose única de 1096 cGy. Essa dose é capaz de produzir alterações anátomo-patológicas na parede intestinal de ratos da linhagem Wistar, cujo efeito máximo é encontrado entre o $7^{\circ}$ e o $14^{\circ}$ dia pósirradiação ${ }^{(10-12)}$.

Para serem utilizados os métodos estereológicos, os cortes analisados devem ser isotrópicos, ou seja, os diversos elementos que o constituem devem estar distribuídos ao acaso e homogeneamente no espaço ${ }^{13-}$ ${ }^{16}$. Dessa forma qualquer corte bidimensional, aleatório e uniformemente isotrópico, passa a ser representativo de um todo tridimensional, independentemente da direção em que esse corte seja realizado.

A parede colônica não é um meio isotrópico, pois possui estruturas de orientação definida, como é o caso das criptas de Lieberkühn, que apresentam disposição paralela entre si e são perpendiculares à superfície mucosa. Para contornar o problema da anisotropia de estruturas como a parede colônica, foi proposto o método de estereologia de cortes verticais ${ }^{17}$, na qual os cortes histológicos são perpendiculares a uma superfície horizontal, representada por um plano existente na estrutura em estudo ou criada arbitrariamente pelo pesquisador.
Neste estudo, foi considerado como plano horizontal o limite entre a mucosa e a submucosa. Esse método permite a compensação da anisotropia e a realização de uma análise estereológica confiável.

O grupo $I+A n$ apresentou mediana da pressão de ruptura da parede colônica significativamente menor do que a do grupo An no sétimo dia pósoperatório. Outros estudos utilizando radioterapia intra-operatória ${ }^{18}$ e radioterapia externa préoperatória ${ }^{(19)}$ encontraram resultados semelhantes. No entanto alguns autores ${ }^{20-21}$ não encontraram diferença estatisticamente significativa na pressão de ruptura da parede colônica entre os grupos controle e irradiado. A comparação destes últimos trabalhos com o presente estudo torna-se prejudicada pois um deles utilizou intervalos de tempo diferentes entre a irradiação e a operação, enquanto o outro utilizou irradiação fracionada.

O local mais freqüente de ruptura da parede colônica no sétimo dia pós-operatório no grupo $A n$ foi a região perianastomótica, enquanto que no grupo $I+$ An essa ruptura ocorria na linha anastomótica. Esses resultados estão de acordo com dados obtidos por outros autores ${ }^{(19)}$ e poderia ser justificado pela diminuição do índice de colágeno (IC) na área da anastomose $^{9}$.

O índice de colágeno na área da anastomose diminuiu no grupo $I+A n$ quando comparado ao grupo $A n$. Essa observação está de acordo com os resultados de outros estudos que encontraram diminuição do colágeno na área de anastomose em ratos que receberam irradiação pré-operatória ${ }^{18}$ ou da quantidade de hidroxiprolina, o principal aminoácido componente do colágeno, na parede do cólon em ratos irradiados ${ }^{19}$.

Alguns autores ${ }^{22}$ identificaram uma redução precoce e significativa do fluxo sanguíneo nas camadas serosa e muscular, em anastomoses colorretais realizadas em um modelo experimental de irradiação pré-operatória. Demonstraram ainda que essa redução era independente da técnica de anastomose utilizada.

A atividade da enzima mieloperoxidase pode ser usada como um marcador do acúmulo de leucócitos na área da anastomose ${ }^{23}$ e pode estar significativamente diminuída em anastomoses feitas em colón irradiado ${ }^{19}$. Esse achado pode significar uma redução significativa no número de leucócitos na área de cicatrização, principalmente de macrófagos, que 
são de importância fundamental no processo de cicatrização. Além disso, a diminuição do fluxo sanguíneo na área de anastomose, pode ser um fator limitante ao aporte de citocinas endócrinas e nutrientes, essenciais à síntese de colágeno ${ }^{5}$.

\section{Conclusões}

A irradiação causa diminuição da tensão de ruptura da parede colônica e da quantidade de colágeno na área da anastomose, quando comparados a animais não irradiados. A ruptura da parede colônica ocorre com maior freqüência nos segmentos adjacentes à anastomose em animais não irradiados, contrastando com os que receberam irradiação, nos quais a ruptura é mais freqüente na linha anastomótica.

\section{Referências}

1. Anseline PF, Lavery IC, Fazio VW. Radiation injury of the rectum: evaluation of surgical treatment. Ann Surg 1981; 194: 716-24.

2. Keighley MRB, Williams NS. Surgery of the anus, rectum and colon. London: W.B. Saunders; 1993.

3. Rider WD, Palmer JA, Mahoney LJ, Robertson CT. Preoperative irradiation in operable cancer of the rectum: report of the Toronto trial. Can J Surg 1977; 20: 335-8.

4. Stevens KR Jr, Fletcher WS, Allen CV. Anterior resection and primary anastomosis following high dose preoperative irradiation for adenocarcinoma of the rectosigmoid. Cancer 1978; 41:2065-71.

5. Cohen IK, Diegelmann RF, Crossland MC. Wound care and wound healing. In: Schwartz SI, Shires GT, Spencer FC. Principles of surgery. 6ed. New York: McGraw-Hill; 1994. p. 279-303.

6. Lopes-Paulo F. Efeitos da L-glutamina na parede do cólon derivado: estudo estereológico experimental em ratos [Tese]. Universidade Federal do Rio de Janeiro; 1999.

7. Lopes-Paulo F. Emprego da estereologia em pesquisas colorretais. Rev Bras Coloproct 2002; 22: 73-6.

8. Lopes-Paulo F. Effects of oral supplement of L-glutamine on diverted colon wall. J Cell Mol Med 2002; 6: 377-82.

9. Lopes-Paulo F. Efeitos da L-glutamina em anastomoses realizadas em cólon irradiado: estudo manométrico e estereológico em ratos [Tese]. Universidade do Estado do Rio de Janeiro - Faculdade de Ciências Médicas; 2003.

10. Northway MG, Scobey MW, Geisinger KR. Radiation proctitis in the rat. Sequential changes and effects of anti-inflammatory agents. Cancer 1988; 62: 1962-9.

11. Gurbuz AT, Kunzelman J, Ratzer EE. Supplemental dietary arginine accelerates intestinal mucosa regeneration and enhances bacterial clearance following radiation enteritis in rats. J Surg Res 1988; 74: 149-54.

12. Ersin S, Tuncyurek P, Esassolak M, Murat A, Buke C, YIlmaz M, Telefonku A, Kose T. The prophylactic and therapeutic effects of glutamine and arginine enriched diets on radiation-induced enteritis in rats. J Surg Res 2000; 89: 121-5.

13. Delesse M. Procédé mécanique pour determiner la composition des roches. Ann Mines 1848; 13:379-88.

14. Chalkley HW, Cornfield I, Park H. A method for estimating volume-surface ratios. Science 1949; 110: 295-7.

15. Weibel ER, Gomez DM. A principle for counting tissue structures on random sections. J Appl Phisiol 1962; 17: 343-8.

16. Elias H, Hyde DM. A guide to practical stereology. Basiléia: Karger; 1983.

17. Baddeley AJ, Gundersen HJG, Cruz-Orive LM. Estimation of surface area from vertical sections. J Microsc 1986; 142: 259-76.

18. Seifert WF, Wobes T, Hoogenhout J, de Man BM, Huyben KM, Hendriks T. Intraoperative irradiation delays anastomotic repair in rat colon. Am J Surg 1995; 170:256-61.

19. Kuzu MA, Koksoy C, Akyol FH, Uzal D, Kale T, Demirpence E. Effects of preoperative fractionated irradiation on left colonic anastomoses in the rat. Dis Colon Rectum 1998; 41: 370-6.

20. Weiber S, Jiborn H, Zederfeldt B. Preoperative irradiation and colonic healing. Eur J Surg 1994; 160: 47-51.

21. Biert J, Hoogenhout J, Wobbes T, Hendriks T. Highdose preoperative irradiation without detrimental effect on early repair of anastomoses in the colon of the rat. Radiat Res 1997; 147: 362-8.

22. Milson JW, Senagore A, Walshal RK. Preoperative radiation therapy produces an early and persistent reduction in colorectal anastomotic blood flow. J Surg Res 1992; 53: 464-9.

23. Suzuki K, Ota H, Sasagawa S, Sakatani T, Fugikura T. Assay method for myeloperoxidase in human polymorphonuclear leucocytes. Anal Biochem 1983; 132: 345-52.

Correspondência:

Francisco Lopes-Paulo

Hospital Universitário Pedro Ernesto

Disciplina de Coloproctologia

Av. 28 de Setembro, 77/4 andar

20551-030 Rio de Janeiro - RJ

fpaulo@lampada.uerj.br 
Lopes-Paulo F

Recebimento: 12/06/2004

Revisão: 08/07/2004

Aprovação: 18/08/2004

Conflito de interesse: nenhum

Fonte de financiamento: nenhuma

\section{Como citar este artigo:}

Lopes-Paulo F. Efeitos da irradiação com raios-X em anastomoses colônicas: estudo estereológico experimental em ratos. Acta Cir Bras [serial online] 2004 Set-Out;19(5). Disponível em URL: http://www.scielo.br/acb [também em CD-ROM].

* Figuras coloridas disponíveis em www.scielo.br/acb 